\title{
The Information Coding in the Time Structure of the Object of a Laser Pulse in an Optical Echo Processor
}

\author{
L. A. Nefediev and A. R. Sakhbieva \\ Kazan (Volga Region) Federal University, 18 Kremliovskaya Street, Kazan 420021, Russia \\ Correspondence should be addressed to A. R. Sakhbieva, alsu-sakhbieva@yandex.ru \\ Received 4 April 2012; Accepted 2 June 2012 \\ Academic Editor: Kiyoshi Shimamura
}

Copyright ( $) 2012$ L. A. Nefediev and A. R. Sakhbieva. This is an open access article distributed under the Creative Commons Attribution License, which permits unrestricted use, distribution, and reproduction in any medium, provided the original work is properly cited.

The encoding of information in time intervals of an echelon of laser pulses of an object pulse in the optical echo processor is considered. The measures of information are introduced to describe the transformation of classical information in quantum information. It is shown that in the description of information transformation into quantum information, the most appropriate measure is a measure of quantum information based on the algorithmic information theory.

\section{Introduction}

The methods of dynamical echo holography allow processing and storage of the information, which is carried by object laser pulses. They have prospects on creation of high-speed optical echo-processors [1]. In this case, the information can be incorporated in the amplitude and temporal shape of the exciting laser pulses, in their wave fronts and polarization and also in echelons of laser pulses. Demonstration of frequency-selective optical memory, where the data recording and processing of data occurs both in the time domain and in the frequency slot, is described in [2]. The echoprocessor based on use of long-lived photon echo has been proposed in [3]. The design of this processor is given an opportunity to demonstrate the density of information recording and processing about several gigabits $/ \mathrm{cm}^{2}$ in [4], using compression and tension of data signals through a rapid change of the carrier frequency. From the point of view of the information theory, it is possible to present an echo-processor as an information channel with memory and noise, in an input and output of which the information has a classical appearance and inside of the channel quantum. This channel provides the information transmission and transformation between the different moments in time and directions in space. Common channel features are the information rate, throughput rate, and use factor. Attempts to describe the quantum information processes in general relied on the formulae of classical information theory, which was operated with the quantum probabilities, rather than amplitudes. In [5] it has been shown that the von Neumann entropy has the information and theoretical value asymptotically characterizing the minimal quantum resources required to describe the ensemble of quantum states. This suggests that the enhanced information theory should be defined as a theory which takes into account the quantum phases explicitly. For example, the theory, developed in [6], describes a quantum system, divided into many parts, using only density matrix and von Neumann entropy. This theory includes the Shannon theory as a special case, but also describes quantum entanglement and establishing the correspondence between classical and quantum information in this way. We consider the system (the message), described by the variables $A$ (classical or quantum), and construct its classical and quantum description at the same time. In classical information theory the entropy of Shannon for $A$ is defined as:

$$
J_{c}=-\sum_{n} p(a) \log _{2} p(a)
$$

where the $A$ takes the value a with probability $p(a)$. This entropy can be interpreted as uncertainty about $A$. 
The quantum analogue is the von Neumann entropy (where the subsystem $A$ is described by the density operator $\rho_{A}$ ),

$$
J_{f n}=-\operatorname{Tr}_{A}\left[\rho_{A} \log _{2} \rho_{A}\right]
$$

where $\operatorname{Tr}_{A}$ denotes the trace over degrees of freedom subsystem $A$.

The von Neumann entropy reduces to Shannon entropy, if $\rho_{A}$ is the mixed state, decomposed in orthogonal quantum state.

In the case of echo-processor information reproduction depends on coding method. It is obvious that in this case it will be the best information reproduction if the information is coded in time intervals of the laser pulses echelon of an object pulse.

In the given work we will consider the process of transformation of the classical information put in time intervals of an echelon of laser pulses in quantum at their resonant interaction with two level quantum system.

\section{Von Neumann Entropy in the Description of Systems with Coherent Superposition of Basis States}

Expression of the von Neumann entropy for a two-level system can be represented by means of functions from matrixes

$$
\begin{aligned}
J_{f n}(\rho) & \\
= & -\operatorname{Tr}\left(\rho \log _{2} \rho\right)=\frac{1}{\sqrt{\left(\rho_{11}-\rho_{22}\right)^{2}+4\left|\rho_{12}\right|^{2}}} \\
\times & {\left[2\left|\rho_{12}\right|^{2} \log _{2} \frac{\lambda_{2}}{\lambda_{1}}\right.} \\
+ & \frac{1}{2} \rho_{11}\left(\rho_{22}-\rho_{11}-\sqrt{\left(\rho_{11}-\rho_{22}\right)^{2}+4\left|\rho_{12}\right|^{2}}\right) \log _{2} \lambda_{1} \\
& -\frac{1}{2} \rho_{11}\left(\rho_{22}-\rho_{11}+\sqrt{\left(\rho_{11}-\rho_{22}\right)^{2}+4\left|\rho_{12}\right|^{2}}\right) \log _{2} \lambda_{2} \\
+ & \frac{1}{2} \rho_{22}\left(\rho_{11}-\rho_{22}-\sqrt{\left(\rho_{11}-\rho_{22}\right)^{2}+4\left|\rho_{12}\right|^{2}}\right) \log _{2} \lambda_{1} \\
& \left.-\frac{1}{2} \rho_{22}\left(\rho_{11}-\rho_{22}+\sqrt{\left(\rho_{11}-\rho_{22}\right)^{2}+4\left|\rho_{12}\right|^{2}}\right) \log _{2} \lambda_{2}\right],
\end{aligned}
$$

where

$$
\lambda_{1,2}=\frac{\rho_{11}+\rho_{22}}{2} \pm \frac{1}{2} \sqrt{\left(\rho_{11}-\rho_{22}\right)^{2}+4\left|\rho_{12}\right|^{2}} .
$$

In the absence of coherence in the system from (3) follows

$$
\lim _{\left|\rho_{12}\right| \rightarrow 0} J_{f n}(\rho)=-\rho_{11} \log _{2} \rho_{11}-\rho_{22} \log _{2} \rho_{22} .
$$

As the $\rho_{11} \leq 1$ and $\rho_{22} \leq 1$ then $J_{f n}(\rho) \geq 0$. In the case of a pure state $J_{f n}(\rho) \rightarrow 0$.
The more appropriate measure of quantum information in the presence of coherence in the system can be $K$ complexity and the applications of algorithmic information theory to the description of the quantum information processes $[7,8]$.

\section{Quantum Structural Information in the Medium with Phase Memory}

Since the structural information in the resonant medium is carried by transitional dynamic gratings, which are described by a density matrix, the structural information is contained in the amplitude and phase structure of the density matrix $\rho$. If we match a graph $G$ to this matrix, the measure of structural information will be defined as the measure of the structure uncertainty of this graph [8]. In the simplest case, this uncertainty can be determined by enumerating the corresponding diagrams of the graph. However, the resulting measure of information is incomplete, since it ignores off-diagonal elements of the corresponding matrix of conditional transitions. We will therefore use the notion of $K$-complexity to quantify structural information.

We consider a graph $G$, corresponding to the density matrix of the system. Its elements belong to a finite set $\epsilon$ $V(G)$, consisting of $N$ labeled vertices and $q$ edges, which correspond to the diagonal and off-diagonal elements of the density matrix, respectively. Thus, $V=\Gamma \bigcup Q$ where $\Gamma$ is the set of the vertices of the graph and $Q$ is the set of its edges.

The relative complexity $K$ of the object $G$ is the minimum length $l(p)$ of a program $p$ that can derive object $G_{o}$ from $G$. We define the amount of structural information of $G$ relative to $G_{o}$ as

$$
J=K\left(G, G_{o}\right)-K\left(G_{o}\right) .
$$

We will partition the algorithmic process of deriving object $G_{o}$ from object $G$ into separate steps of bounded complexity. Each step transforms the current state $G^{\kappa}$ of the object to state $G^{\kappa+1}$

$$
G^{\kappa+1}=D_{\kappa}\left(G^{\kappa}\right)
$$

Operator $D$ is determined by the set of rules for processing the active part of object $G$. Thus, we have

$$
G_{o}=D(G)
$$

Since the quantum information lies in the coherent part of the density matrix, we will consider the elements $\in Q$ as the active part object $G$. Operator $D$ is defined as the operator of removing elements from the active part of object $G$ by any possible means [9]:

$$
D(Q)=\oslash \text {. }
$$

We will assume that object with the elements, belonging to the zero set $\oslash$, has structural information, which is equal to 0 . It is convenient to quantify the length $l(p)$ of program $p$ on a logarithmic scale as $\log _{2}$ of the sum of all weighting functions corresponding to diagrams of $G^{\kappa}$. Taking 
the temporal evolution into account, we define the weighting function as the sum of the active part of elements of object $G$ at time $t$. The active part of object $G$ corresponds to diagram $G^{\kappa}$, referred to the sum of the active part elements of object $G$ at the initial time instant $t_{o}$. Procedure (9) leads to an ensemble of sets $Q^{(\kappa)}$. Since weighted graph $G$ corresponds to the density matrix

$$
\rho=\sum_{i, j=1}^{N} \rho_{i j} P_{i j},
$$

where $P_{i j}$ are the projector matrices (which have element $i j$ equal to 1 and all other elements equal to 0 ), then the sum $S\left(t_{o}\right)$ of the active part elements of the object is given at the initial time by

$$
S\left(t_{o}\right)=a b s\left(\sum_{i \neq j} \rho_{i j}\left(t_{o}\right)\right) .
$$

Calculating the corresponding sum $S^{\prime}(t)=\sum_{\kappa} S^{(\kappa)}(t)$ for the ensemble of sets $Q^{(\kappa)}$ at time $t$, we arrive at:

$$
J_{q}=\log _{2}\left(\frac{S^{\prime}(t)}{S\left(t_{o}\right)}\right) .
$$

Since $\hat{\rho}$ is a Hermitian operator, its matrix elements meet the equality $\rho_{i j}=\rho_{j i}^{*}$. Now it is easy to see that our choice of the operator $D$ brings us to $S^{\prime}(t)$ and $S\left(t_{0}\right)$, consisting of matrix elements sum $\rho_{i j}+\rho_{j i}$. Thus, $S^{\prime}(t)$ and $S\left(t_{o}\right)$ are real quantities. We should note also that, when the system has only two quantum states, $|1\rangle$ and $|2\rangle$, the total state is a linear superposition $|\psi\rangle=\alpha|1\rangle+\beta|2\rangle$ (where $\alpha$ and $\beta$ are complex numbers). The relevant density matrix operator is written as

$$
\begin{aligned}
\hat{\rho}= & |\psi\rangle\left\langle\left.\psi|=| \alpha\right|^{2} \mid 1\right\rangle\left\langle\left. 1|+| \beta\right|^{2} \mid 2\right\rangle\langle 2| \\
& +\alpha \beta^{*}|1\rangle\left\langle 2\left|+\alpha^{*} \beta\right| 2\right\rangle\langle 1| .
\end{aligned}
$$

\section{The Transformation Process of the Classical Information in Quantum Structural Information}

We consider the transformation of the classical information $J_{c}(A)$ carried by an object laser pulse during its interaction with a system of two-level atoms, where the quantum information $J_{q}$ carriers which are superposition states of atoms.

Object pulse can be represented as a sequence (echelon) of the $n$ rectangular laser pulses (in the general case with different duration and amplitude $\varepsilon_{\eta}$ ), separated by arbitrary time intervals. Time intervals are denoted as $\tau_{\eta}(\eta=$ $1, \ldots, n)$. Then $\varepsilon_{\eta}>0$ is the presence of a pulse, and $\varepsilon_{\eta}=$ 0 is the presence of a time interval. The duration of the pulse echelon will be $\delta t=\sum \tau_{\eta}$, satisfying $\delta t \ll T_{1}, T_{2}$, where $T_{1}$ and $T_{2}$ are the times of longitudinal and transverse irreversible relaxation of the system considered.

To describe the process of transformating the classical information, into quantum information the most appropriate definition of classical information can be a differential information entropy of the Fourier spectrum of the echelons of laser pulses, because in the resonant medium information carriers are $q$-bits, which are distributed in the range of the inhomogeneously broadened line of the resonance transition.

The intensity of Fourier components of the pulse echelon electric field will be:

$$
E\left(\nu^{\prime}\right)=\sum_{\eta=1}^{n} \varepsilon_{\eta} \int_{t_{\eta-1}}^{t_{\eta}} e^{-i 2 \pi v^{\prime} t} d t,
$$

where $v^{\prime}$ is the frequency of the Fourier spectrum, and the time $t_{\eta}$ of the $\eta$ th pulse start is defined as

$$
t_{\eta}=t_{o}+\sum_{\kappa=1}^{\eta} \tau_{\kappa}
$$

and it is assumed that the initial time $t_{o}=0$. Then from (16) for the amplitude of the Fourier components of the pulse echelon electric field we obtain

$$
A\left(\omega^{\prime}\right)=\left|E\left(\omega^{\prime}\right)\right|=\sqrt{\operatorname{Re}\left(E\left(\omega^{\prime}\right)\right)^{2}+\operatorname{Im}\left(E\left(\omega^{\prime}\right)\right)^{2}},
$$

where $\omega^{\prime}=2 \pi v^{\prime}$,

$$
\begin{aligned}
& \operatorname{Re}\left(E\left(\omega^{\prime}\right)\right)=\sum_{\eta=1}^{n} \varepsilon_{\eta} \tau_{\eta} \operatorname{sinc}\left(\frac{\omega^{\prime} \tau_{\eta}}{2}\right) \cos \left(\frac{\omega^{\prime}\left(2 \sum_{\kappa=1}^{\eta} \tau_{\kappa}-\tau_{\eta}\right)}{2}\right), \\
& \operatorname{Im}\left(E\left(\omega^{\prime}\right)\right)=\sum_{\eta=1}^{n} \varepsilon_{\eta} \tau_{\eta} \operatorname{sinc}\left(\frac{\omega^{\prime} \tau_{\eta}}{2}\right) \sin \left(\frac{\omega^{\prime}\left(2 \sum_{\kappa=1}^{\eta} \tau_{\kappa}-\tau_{\eta}\right)}{2}\right) .
\end{aligned}
$$

Differential information entropy of Fourier spectrum of the laser pulses echelon is defined as $J_{c}^{\prime}=J_{c}-J_{c 0}$, where

$$
J_{c}=-\int_{-\infty}^{\infty} p\left(\omega^{\prime}\right) \log _{2} p\left(\omega^{\prime}\right) d \omega^{\prime} .
$$

There

$$
p\left(\omega^{\prime}\right)=\frac{A\left(\omega^{\prime}\right)}{\int_{-\infty}^{\infty} A_{o}\left(\omega^{\prime}\right) d \omega^{\prime}},
$$

and $A_{o}\left(\omega^{\prime}\right)$ is determined from the expression (17), $J_{c 0}$ is defined similarly to (19) at identical amplitudes and time intervals in (17) and (18).

To find the values of quantum information (algorithmic or von Neumann) it is necessary to calculate the density matrix of the resonant system after exposure to the object pulse (echelon).

Neglecting power broadening and spectral diffusion during the object pulse we find the density matrix in the interaction of the atom with a single Fourier component of the pulse echelon field with subsequent averaging over all frequencies.

The electric intensity of Fourier components of the pulse field can be written as

$$
E\left(\omega^{\prime}\right)=\frac{1}{2}\left[E^{*}\left(\omega^{\prime}\right) e^{i\left(\omega-\omega^{\prime}\right) t}+E\left(\omega^{\prime}\right) e^{-i\left(\omega-\omega^{\prime}\right) t}\right] .
$$



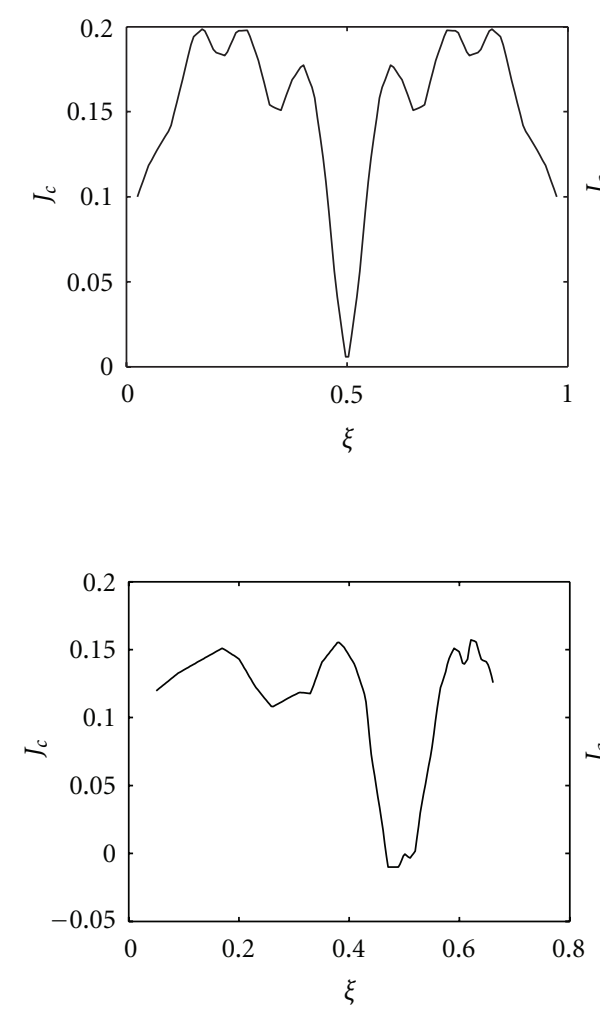
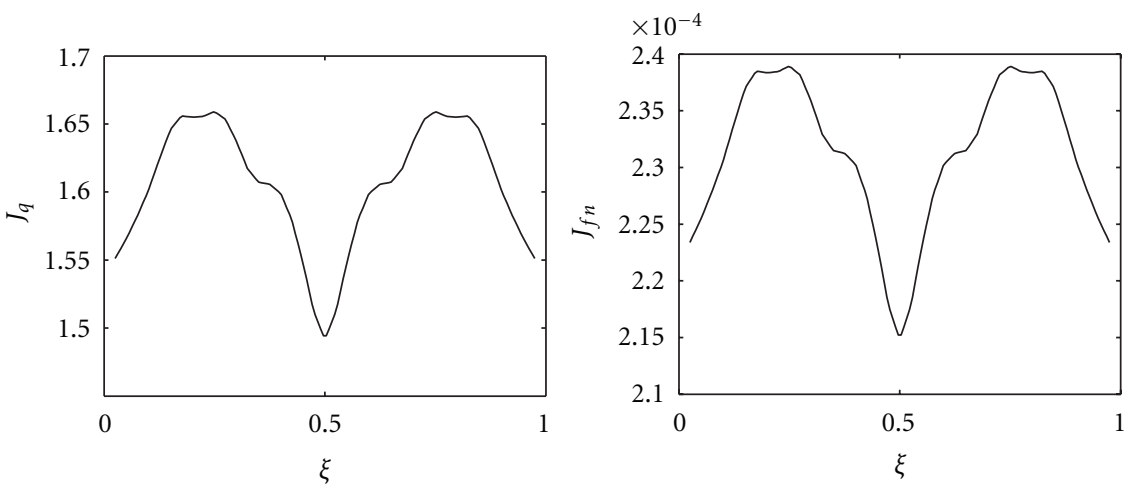

(a)
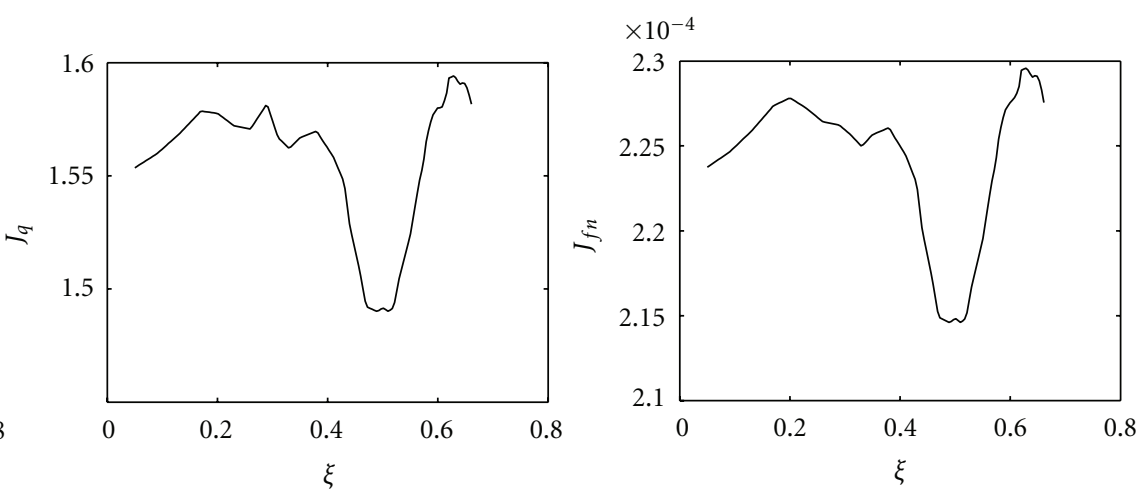

(b)

FIGURE 1: (a) Information measures in case of position change of the middle pulse in the object pulse echelon. (b) Information measures in case of position change of the extreme pulse in the object pulse echelon. $\xi=\tau_{1} /\left(\tau_{1}+\tau_{2}\right) ; \theta=\pi / 2$ : area of the object pulse; $n=\sigma \cdot \delta$ where $\sigma$ is a half-width of the inhomogeneously broadened line, $\delta t$-the pulse echelon duration. $J_{c}$ : classical information; $J_{q}:$ quantum information; $J_{f n}$ : von Neumann entropy.

The frequency of the Fourier spectrum $\omega^{\prime}$ can be either $>0$ or $<0$. The frequency of the transition in an atom $=\Omega$, but in the case of interaction with the local fields (crystal), it becomes $\Omega-\Omega^{\prime}$, where $\Omega^{\prime}$ is the value of frequency shift. $\Omega^{\prime}$ can be either $>0$ or $<0$ within the inhomogeneously broadened line of the atomic transition. We assume that $\omega=\Omega$, that is, the central laser frequency coincides with the transition frequency in the absence of the local field.

In this case, the equation for the Fourier components of the single-particle density matrix can be written as

$$
\frac{\partial \tilde{\rho}}{\partial t}=-\frac{i}{\hbar}[B, \tilde{\rho}]
$$

where

$$
\begin{gathered}
B=\tilde{H}_{0}-\hbar A+\widetilde{V}, \\
H_{0}=\hbar\left(\Omega-\Omega^{\prime}\right) P_{22}, \quad A=\left(\omega-\omega^{\prime}\right) P_{22}, \\
e^{ \pm i A t}=P_{11}+P_{22} e^{ \pm i\left(\omega-\omega^{\prime}\right) t}, \\
\tilde{V}=-\frac{1}{2} d\left[E^{*}\left(\omega^{\prime}\right) P_{12}+E\left(\omega^{\prime}\right) P_{21}\right],
\end{gathered}
$$

$d$ is the dipole moment of the resonance transition, $P_{i j}$ are the projector matrices (which have element $i j$ equal to 1 and all other elements equal to 0 )

$$
B=\left(\begin{array}{cc}
0 & -\frac{1}{2} d E^{*}\left(\omega^{\prime}\right) \\
-\frac{1}{2} d E\left(\omega^{\prime}\right) & \hbar\left(\omega^{\prime}-\Omega^{\prime}\right)
\end{array}\right) .
$$

The solution of (22) can be written as

$$
\tilde{\rho}(t)=e^{-i \hbar^{-1} B t} \rho(0) e^{i \hbar^{-1} B t} .
$$

The bordering exponents in (25) can be determined by methods of matrix functions. In the case $t=0 \rho_{22}(0) \rightarrow 0$, $\rho_{11}(0) \rightarrow 1$ and

$$
\begin{aligned}
\tilde{\rho}(t) \approx & P_{11}\left(\cos ^{2} \frac{\theta}{2}+\frac{\Delta^{2}}{\theta^{\prime 2}} \sin ^{2} \frac{\theta}{2}\right) \\
& +P_{12}\left(-i \frac{a^{*}}{2 \theta^{\prime}} \sin \theta+\frac{a^{*} \Delta}{\theta^{\prime 2}} \sin ^{2} \frac{\theta}{2}\right) \\
& +P_{21}\left(i \frac{a}{2 \theta^{\prime}} \sin \theta+\frac{a \Delta}{\theta^{\prime 2}} \sin ^{2} \frac{\theta}{2}\right)+P_{22} \frac{|a|^{2}}{\theta^{\prime 2}} \sin ^{2} \frac{\theta}{2}
\end{aligned}
$$


where

$$
\begin{gathered}
\Delta=\omega^{\prime}-\Omega^{\prime}, \quad \theta=\theta^{\prime} t, \quad \theta=\sqrt{\Delta^{2}+d^{2} E_{0}^{2} \hbar^{-2}|\tilde{\mathcal{\varepsilon}}|^{2}}, \\
a=d E_{0} \hbar^{-1} \widetilde{\mathcal{E}} e^{i \vec{k} \vec{r}}, \quad a^{*}=d E_{0} \hbar^{-1} \widetilde{\mathcal{E}}^{*} e^{i \vec{k} \vec{r}} .
\end{gathered}
$$

After pulse excitation $B=P_{22} \hbar \Delta$, that is

$$
\begin{gathered}
e^{ \pm i \hbar^{-1} B\left(t^{\prime}-t\right)}=P_{11}+P_{22} e^{ \pm i \Delta \cdot\left(t^{\prime}-t\right)} \\
\tilde{\rho}\left(t^{\prime}-t\right)=\left(P_{11}+P_{22} e^{-i \Delta \cdot\left(t^{\prime}-t\right)}\right) \tilde{\rho}(t)\left(P_{11}+P_{22} e^{i \Delta \cdot\left(t^{\prime}-t\right)}\right)
\end{gathered}
$$

The final result for the quantum algorithmic information is of the form

$$
J_{q}=\int_{-\infty}^{\infty} p\left(\omega^{\prime}\right) d \omega^{\prime} \int_{-\infty}^{\infty} g\left(\Omega^{\prime}\right) J_{q}\left(\omega^{\prime}, \Omega^{\prime}\right) d \Omega^{\prime},
$$

where $g\left(\omega^{\prime}\right)$ is the frequency distribution of the inhomogeneously broadened line of the resonant transition, and $J_{q}\left(\omega^{\prime}, \Omega^{\prime}\right)$ is defined by the expression (12).

By analogy, for the von Neumann entropy we have

$$
J_{f n}=\int_{-\infty}^{\infty} p\left(\omega^{\prime}\right) d \omega^{\prime} \int_{-\infty}^{\infty} g\left(\Omega^{\prime}\right) J_{f n}\left(\rho\left(\omega^{\prime}, \Omega^{\prime}\right)\right) d \Omega^{\prime},
$$

where $J_{f n}\left(\rho\left(\omega^{\prime}, \Omega^{\prime}\right)\right)$ is defined by the expression (3).

After the influence on the resonant medium of the object pulse that carries classical information, this information is distributed over separate isochromatic components of the inhomogeneously broadened line. In other words, an information-phase grating within the inhomogeneously broadened line of the resonant transition arises. Every single $q$-bit can have a piece of classical (the diagonal part of the density matrix) and quantum information (off-diagonal part of the density matrix).

In case when the information lies in the time intervals of the laser pulse echelon, the minimum structure, which carries information, is a sequence of three laser pulses with unequal time intervals $\tau_{1}$ and $\tau_{2}$ between them. For such a structure the conversion $J_{c} \rightarrow J_{q}$ result is shown in Figure 1 .

Figure 1(a) shows the values of information measures in case of the position change of the middle exciting pulse in the object pulse echelon and Figure 1(b) shows the values of information measures in case of the position change of the extreme exciting pulse in the object pulse echelon (the object pulse consists of 3 pulses). The highest obtained correlation coefficient between the classical and quantum information $\approx 0,94$.

\section{Conclusion}

The best classical information measure in the case of information encoding in the time intervals is the differential information entropy of the object laser pulse Fourier spectrum. Quantum information measure, based on algorithmic information theory, is the most appropriate for description of superpositional quantum system. Information encoding in the time intervals in laser pulses' echelon causes minimal information distortions in response to the resonant system.

\section{References}

[1] A. A. Kalachev and V. V. Samartsev, Kogerentnyye yavleniya $v$ optike, Kazan State University, Coherent phenomena in optics, Kazan, Russia, 2003.

[2] M. Mitsunaga, R. Yano, and N. Uesugi, "Time- and frequencydomain hybrid optical memory: 1.6-kbit data storage in $\mathrm{Eu}^{3+}: \mathrm{Y}_{2} \mathrm{SiO}_{5}$," Optics Letters, vol. 16, no. 23, pp. 1890-1892, 1991.

[3] H. Un, T. Wang, G. A. Wilson, and T. W. Mossberg, "Experimental demonstration of swept-carrier time-domain optical memory," Optics Letters, vol. 20, no. 1, pp. 91-93, 1995.

[4] H. Un, T. Wang, and T. W. Mossher, "Demonstration of 8 -Gbit/in. ${ }^{2}$ areal storage density based on swept-carrier frequency-selective optical memory," Optics Letters, vol. 20, no. 15, pp. 1658-1660, 1995.

[5] B. Schumacher, "Quantum coding," Physical Review A, vol. 51, no. 4, pp. 2738-2747, 1995.

[6] N. J. Cerf and C. Adami, "Negative entropy and information in quantum mechanics," Physical Review Letters, vol. 79, no. 26, pp. 5194-5197, 1997.

[7] L. A. Nefed'ev and I. A. Rusanova, "Copying quantum information in a three-level medium with a phase memory," Laser Physics, vol. 12, no. 3, pp. 571-575, 2002.

[8] A. N. Kolmogorov, Teoriya informatsii $i$ teoriya algoritmov, Nauka, Moscow, Russia, 1987.

[9] L. A. Nefed'ev and I. A. Rusanova, "Information processes in optical echo holography," Optics and Spectroscopy, vol. 90, no. 6, pp. 906-910, 2001. 

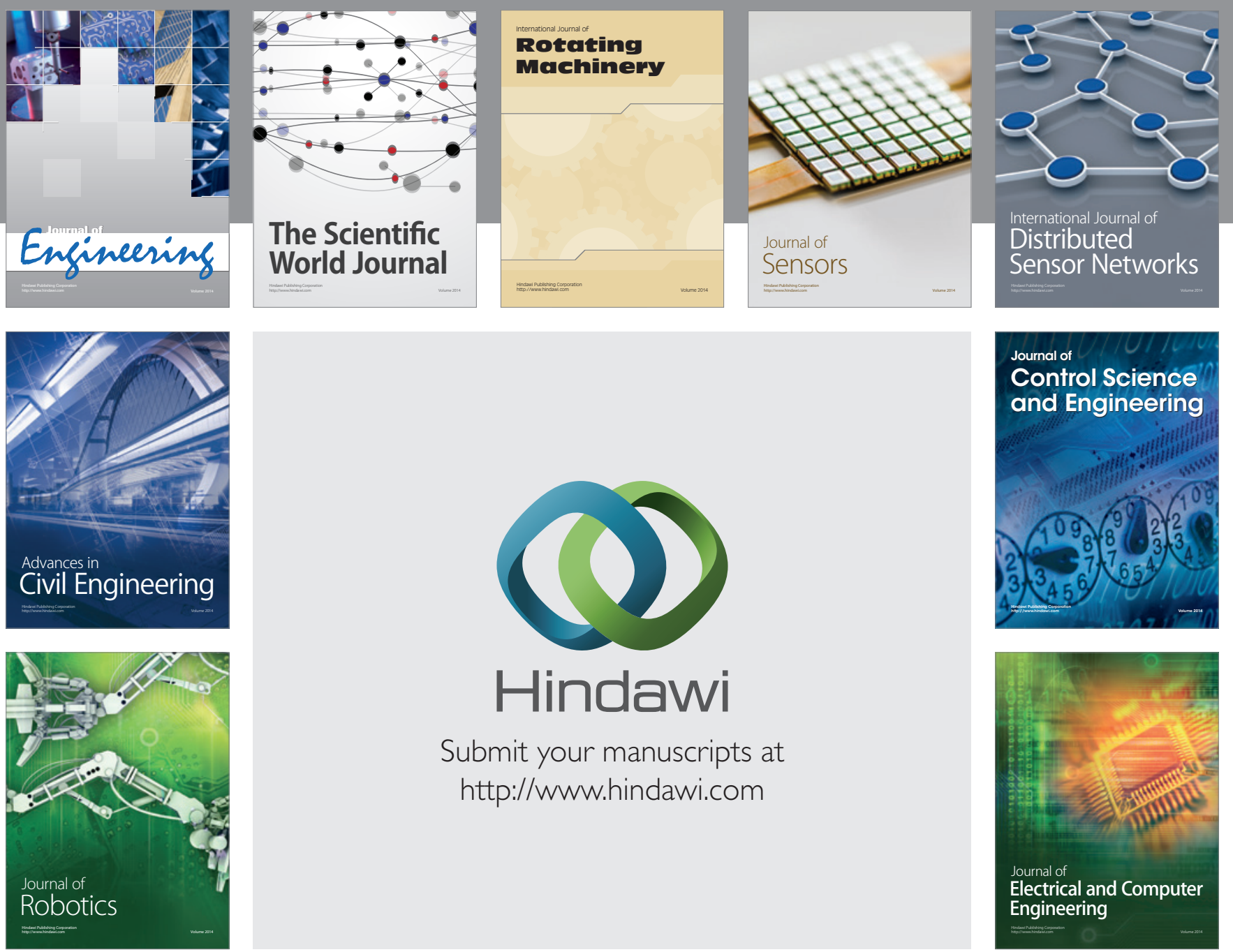

Submit your manuscripts at

http://www.hindawi.com
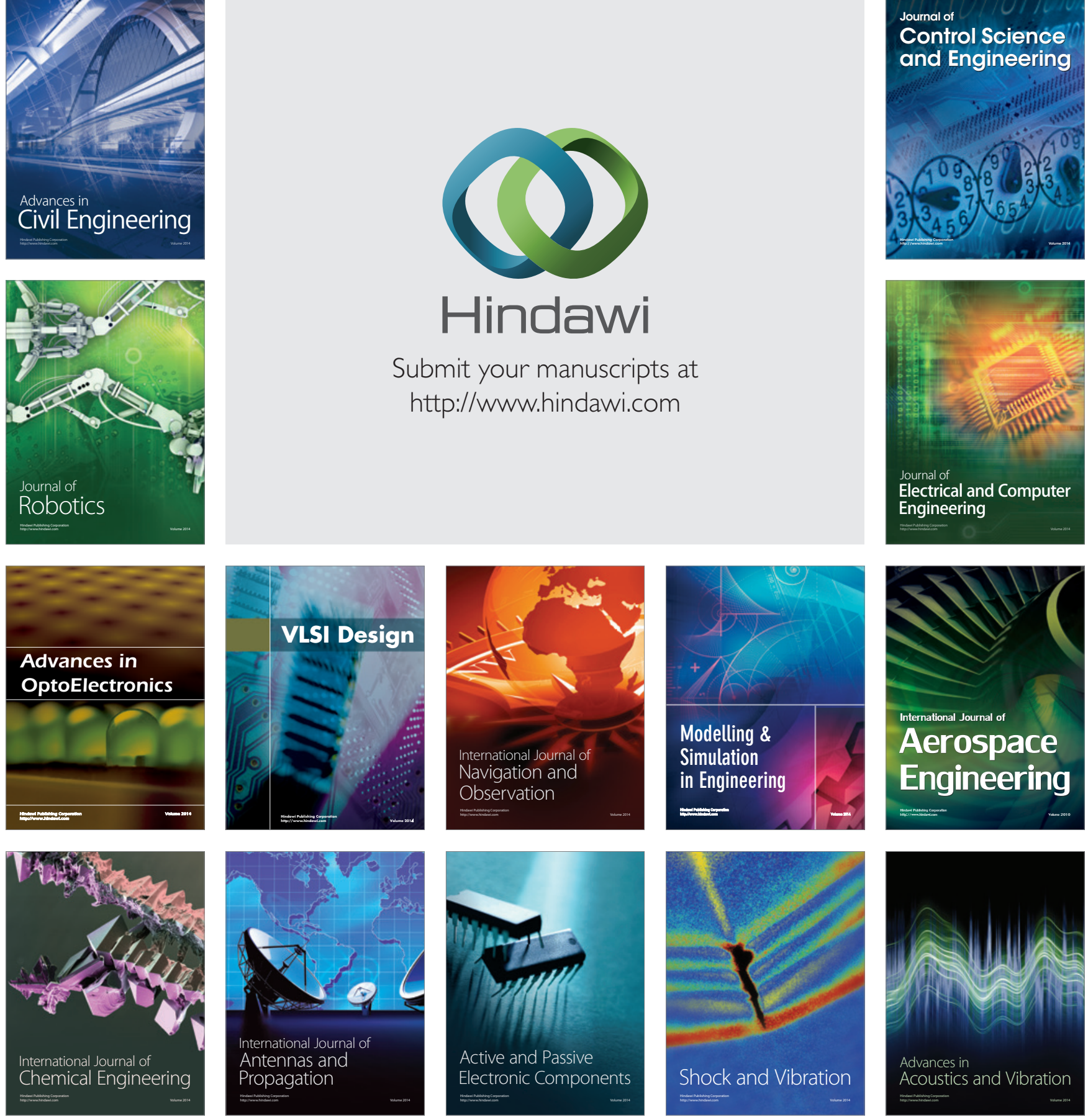